Las Nadies: trabajadoras invisibilizadas. Análisis de discursos sobre las "jubilaciones de amas de casa" en medios gráficos nacionales Tatiana Marisel Pizarro

Question, Vol. 1, N.ํ 56, e013, octubre-diciembre 2017. ISSN 1669-6581

http://perio.unlp.edu.ar/ojs/index.php/question/article/view/4430

FPyCS | Universidad Nacional de La Plata

La Plata | Buenos Aires | Argentina

Recibido: 20-10-2017 Aceptado: 14-11-2017

Cita sugerida: Pizarro, T. (2017). Las Nadies: trabajadoras invisibilizadas. Análisis de discursos sobre las "jubilaciones de amas de casa" en medios gráficos nacionales. Question, 1(56), e013. doi: https://doi.org/10.24215/16696581e013

\title{
Las Nadies: trabajadoras invisibilizadas. Análisis de discursos sobre las "jubilaciones de amas de casa" en medios gráficos nacionales
}

\author{
Las Nadies: invisible workers. Discourse analysis about "housewife \\ retirements" in national media
}

Tatiana Marisel Pizarro Instituto de Investigaciones Socio Económicas; Facultad de Ciencias Sociales; Universidad Nacional de San Juan/ Consejo Nacional de Investigaciones Científicas y Técnicas (Argentina) tatianamariselpizarro

\section{Resumen}

El presente artículo es un extracto de la investigación realizada para la tesis de Maestría en Políticas Sociales de la Universidad Nacional de San Juan. En dicho estudio se indagó sobre las representaciones en medios gráficos nacionales de la figura del ama de casa dentro del sistema previsional argentino, adoptando como eje de análisis una perspectiva de género. Este trabajo plantea interpretar los vínculos entre las informaciones difundidas durante la inserción del ama de casa como beneficiaria del Plan de Inclusión Previsional y el contexto en el que 
ocurrió, por lo que se tratará de distinguir qué propiedades del discurso son utilizadas como formas de interacción controladas por el emisor y utilizadas como manifestaciones de imaginarios colectivos presentes cuando se habla de la tarea del ama de casa en el hogar. Se pretende que este artículo sea una contribución relevante para fortalecer el reconocimiento del trabajo de las mujeres, dé cuenta de la desigualdad que implica su invisibilización y brinde elementos a los medios de comunicación para una correcta perspectiva de género en la difusión de informaciones.

Palabras claves: perspectiva de género; análisis del discurso; medios gráficos.

\section{Abstract}

This article is an extract from their search carried out for the Master's thesis in Social Policies of the Universidad Nacional de San Juan. This study investigated their presentations in national media of the figure of the housewife within the Argentine pension system. It is intent to make an analysis taking a gender's perspective. Thus, this paper proposes to interpret the relations between the information disseminated during the insertion of the housewife as beneficiary of the Social Inclusion Plan and the context in which it occurred, so that it will try to distinguish which properties of the discourse are used as forms of interaction controlled by the sender and used as manifestations of collective imaginaries existents when describe the task of the housewife in the home. It pretends that this paper is a relevant contribution to streng then there cognition of the work of women, account for the inequality that implies their invisibilization and provides elements to the media for a correct gender's perspective in the dissemination of information.

Keywords: gender's perspective; discourse analysis; medias.

Desde las escrituras que plantean que Eva fue creada por Dios de la costilla de Adán-y no de la columna $u$ otro hueso-, la mujer ha sido vista como un ser inferior al hombre por considerarse una parte de él y no su semejante.

A lo largo de la historia el rol de la mujer dentro de la sociedad capitalista ha sido obviado o considerado irrelevante en el trajín del mercado. Adam Smith vio -en el papel de la mujer dentro del hogar- el factor que contrarrestaba el individualismo y el materialismo característico del mercado con altruismo y criterios morales. Contrariamente, Marx obvia el trabajo doméstico en su división social del trabajo, reduciéndolo al consumo de mercaderías obtenidas por la venta de la fuerza de trabajo del hombre en la sociedad capitalista. Mientras que Engels y Marshall median con éste. El primero, contrario a Marx, dota de importancia al papel de la 
mujer en cuanto al "cuidado" de la familia -aunque lo mantiene alejado de toda producción social- y plantea la tensión que habría en el hogar ante la demanda del mercado capitalista de incorporar fuerza de trabajo femenino. Marshall le agregó un elemento: un salario familiar para los trabajadores varones -si bien se hablaba de un mínimo se consideraba suficiente para "sostener" a las esposas y así valorar su trabajo altruista-.

Actualmente, a pesar de esta invisibilización, las tareas domésticas y de reproducción realizadas en el interior del hogar contribuyen de manera directa en la persistencia del modo capitalista de producción -otorgando fuerza de trabajo para su venta-. Esta reproducción y reconstrucción de fuerza de trabajo repercute en la generación de bienes y servicios, pero al desarrollarse en el ámbito privado del hogar y fuera de los circuitos de intercambio mercantil, se torna invisible.

Con la feminización de los mercados laborales, gran parte de ese trabajo reproductivo pasó a ser pago por la transferencia de la esfera doméstica al mercado-guarderías, geriátricos, personal doméstico, tintorerías, etcétera-. Es oportuno subrayar que muchos de estos servicios son ofrecidos casi de forma exclusiva por mujeres, pero en este caso estadísticamente visibles. En el momento en que este trabajo reproductivo deja de desarrollarse en esta sociedad de mercado -en la que los valores hegemónicos se asocian al movimiento y la actividad- lleva a quien lo realiza a formar parte del grupo de los inactivos, adquiriendo consecuencias de imagen social y autoidentificación que va más allá de connotaciones económicas (Otegui Pascual, 1999: 135-136).

En el caso de las mujeres -consideradas inactivas a pesar de desarrollar largas jornadas de trabajo no remunerado en el hogar-, esta situación tiene consecuencia directa para su ingreso futuro al sistema previsional. Este sólo prevé protección a las mujeres a través de su marido, por suderecho a una pensión en caso de muerte, siendo esta una cobertura "derivada" de la condición familiar.

Por otro lado, a pesar de la tendencia al alza, aún hoy las mujeres siguen presentando tasas de participación en el mercado laboral menores que los hombres, por lo que a la hora de reclamar un beneficio jubilatorio contributivo las mujeres se encuentran en desventaja: acumulan menos aportes y por lo tanto muchas de ellas no logran alcanzar el mínimo requerido para obtener un beneficio; y las que sí lo obtienen, reciben beneficios más bajos, ya sea por haber contribuido pocos años o porque sus aportes y/o ingresos laborales fueron menores (Arza, 2013).

En las últimas décadas, en América Latina, se impulsaron políticas alineadas a expandir la protección económica de los/as adultos/as mayores mediante prestaciones no contributivas, que no están rigurosamente relacionadas con la existencia de una trayectoria laboral formal.En Argentina, la precarizaciónlaboral, las restricciones fiscales y los cambios en las estructuras familiares vividos en las décadas de 1980 y 1990, impulsaron la creación de políticas y programas que ampliarán la cobertura y el número de beneficiarios/as del sistema de seguridad 
social. Un ejemplo claro de estas medidas es el Plan de Inclusión Previsional -PIP-. Esta política corresponde a una pensión parcialmente no contributiva que buscaba la inclusión al sistema previsional de aquellas personas que no cumplieran con los aportes formales exigidos por la Ley 24.241, contribuyendo en algunos casos a superar situaciones de vulnerabilidad social y de pobreza.

En este sentido, este artículo presentaun estudio sobre las divergencias existentes entre el concepto de trabajo y la valoración del rol del ama de casa como tal, mediante la interpretación de discursos periodísticos emitidos sobre el Plan de Inclusión Previsional, conocido en el decir cotidiano como "jubilación del ama de casa". Se considera, entonces, que esta contribución resulte relevante para fortalecer el reconocimiento del trabajo de las mujeres, dar cuenta de la desigualdad que implica su invisibilización y brindar elementos a los medios de comunicación para una correcta perspectiva de género en la difusión de informaciones.

\section{Enfoque teórico}

La noción de patriarcado desarrollada por la teoría feminista alude a la preeminencia de relaciones de género asimétricas, que subordinan la posición de las mujeres en la estructura económica y social. Esta es una conformación social consolidada en el marco de sociedades capitalistas, en las que el espacio de la producción y la reproducción adquieren límites más precisos (en relación con formas de producción pre-capitalistas donde las acciones de producción y reproducción se fundían tanto en términos espaciales como temporales). Con la industrialización, la unidad doméstica fue separada de la unidad de producción y se estableció una división sexual del trabajo más rígida. Así, la mujer quedó principalmente a cargo de las tareas reproductivas dentro del hogar, en tanto el hombre pasó a desempeñarse en tareas productivas fuera de ese espacio, a cambio de una remuneración monetaria.

En las condiciones actuales, la pretendida decisión de las mujeres de retirarse de la oferta de trabajo para dedicarse de manera principal o única al cuidado de los hijos e hijas representa una acción en la que no sólo pesa el ideal "tradicional" de la división sexual del trabajo, sino también la evaluación acerca de la conveniencia (o no) de participar en un mercado laboral con oportunidades estrechas (o escasamente remuneradas) para mujeres pobres, frente a la escasez de servicios de cuidado gratuitos, así como los riesgos latentes que se asocian al cuidado por parte de personas desconocidas (Pereyra, 2012).

\section{La naturalización del trabajo del cuidado como actividad de la mujer}


La construcción cultural de la división sexual del trabajo naturalizó la responsabilidad de las mujeres en el cuidado de los miembros del hogar. Adicionalmente, el papel de esposa y madre fue mistificado: el que las mujeres se dedicaran sólo al hogar se transformó en un símbolode estatus y se generó un culto a la domesticidad, en el cual la familia y el hogar pasaron a ser considerados espacios de afecto y crianza, a cargo de ellas. De este modo, se racionalizaron dos creencias: la primera sostiene que el trabajo no remunerado en el hogar es un trabajo de mujer y, la segunda, que en realidad no se trata realmente de trabajo (OIT-PNUD, 2009).Si bien las formas de relación y en particular el vínculo de la mujer con el mundo del trabajo remunerado se han ido modificando, estas naturalizaciones persisten.

Al hablar de familia, el imaginario colectivo automáticamente construye la idea del modelo patriarcal, en el que se le dota al hombre el papel de proveedor del grupo (principio de manutención) y a la mujer se le asignan las actividades de cuidado de los miembros de ese grupo (principio de los cuidados domésticos), dotadas de un perfil obligatorio por su "naturaleza femenina", y por lo tanto desvalorizadas. Este trabajo no remunerado se ubica bajo un manto de "invisibilidad" en el reconocimiento del ámbito económico, por la concepción errónea de sólo pertenecer al carácter privado de las relaciones familiares. Estas se reducen a la cultura transmitida, los valores ético-culturales e ideológicos que han construido representaciones sociales sobre feminidad y masculinidad, maternidad, que se traducen en una desigual distribución sexual del tiempo de trabajo, de derechos y acceso a programas y beneficios (Orloff, 1996).Cabe destacar que los valores, normas, pautas de conductas, desarrollo y reproducción son considerados como deber y responsabilidad de la familia en su función de socialización y formador del capital social (Hintze, 2004).

\section{El rol económico del trabajo de cuidado no remunerado}

Sin embargo, el trabajo doméstico debe considerarse como un elemento necesario e imprescindible que influye en ámbitos que van más allá de lo meramente privado, ya que contribuye en forma directa a la persistencia del modo capitalista de producción, proveyéndole de fuerza de trabajo disponible para atender toda su demanda. Existe así un interés común en los hombres en contar con una mujer que esté disponible para servirlos dentro del hogar, resaltando que en el sistema productivo de mercancías no sólo es necesaria la venta de fuerza de trabajo del hombre, sino que es de suma importancia el trabajo de reproducción no remunerado realizado por la mujer. Asimismo, es en buena medida a través del trabajo no remunerado realizado en el ámbito familiar -y por parte de las mujeres- que se compensa y equilibra el déficit que se produce en términos de provisión de servicios por parte del Estado y de la oferta de empleos de calidad por parte de los mercados (Jelin, 2012). Así, el trabajo de cuidado no remunerado se constituye en un elemento esencial a la hora de explicar la manera 
en que las personas acceden al bienestar, a todos los elementos físicos y simbólicos que necesitan para sobrevivir en el marco de las relaciones sociales.

\section{La noción de régimen de bienestar y sus dimensiones de género}

El trabajo de cuidado no remunerado aparece como un elemento clave en el funcionamiento de los regímenes de bienestar (Esping-Andersen, 1990 y 1999) y de la organización social del cuidado (Razavi, 2007). De esta manera, el acceso al bienestar ya no se vincula exclusivamente con la mercantilización o desmercantilización -conceptos propios de EspingAndersen-, sino que se amplía al ámbito doméstico (Martínez Franzoni, 2007).

La llamada "crisis de cuidado" viene a develar las tensiones en las formas diversas de OSC y regímenes de bienestar, todos los cuales -con diferente grado- siguen contando con el trabajo de cuidado no remunerado que realizan las mujeres como un componente dado. Esto repercute directamente en la vida de las mujeres, que enfrentan cargas diversas de responsabilidades domésticas, mediadas por su posición socio-económica. En el marco de esta diversidad, y sujetas a las opciones disponibles para derivar el cuidado a otras personas o a instancias extra-domésticas, las mujeres negocian su trabajo no remunerado con el que sí lo es, desempeñándose desempeñándose en una doble jornada: por un lado, en el ámbito doméstico y, por el otro, en el ámbito doméstico y en una competencia constante en el mercado laboral (CEPAL, 2009). Las políticas de empleo -de desfamiliarización- y hacia la familia -familistas-median entre ambas realidades, con resultados desiguales en el marco de mercados laborales con prevalencia de informalidad y vulnerabilidad laboral (Draibe y Riesco, 2006). Estas políticas producen impactos en los papeles sexuales y en la autonomía de la mujer, tanto en el plano simbólico como en el material. Todo esto es productode un supuesto cultural falso: que el trabajo "natural" no es trabajo, que el tiempo es elástico, y que el trabajo remunerado de la mujer es secundario y, por lo tanto, puede ser peor pagado y más inestable (CEPAL, 2009). La creciente inserción laboral de las mujeres se ha dado en un marco de políticas de conciliación débiles, que como consecuencia negativa ha generado una presión adicional sobre la inversión social, especialmente en contextos de baja prioridad fiscal (OITPNUD, 2009).

\section{Políticas sociales y Sistema de Previsión Social}

Las políticas sociales son la pata principal de la participación del Estado en los regímenes de bienestar. Es necesario precisar que toda política social opera como un organismo pluricelular, cuyas partes se relacionan yconfluyen entre sí. Cada política no es igual a la otra, y el sujeto sobre el que actúa tampoco lo es, lo que determina su singularidad al especificar el tipo de 
política que es.Su existencia es casi axiomática: siempre hay política social, del mismo modo que siempre hay Estado (Danani, 2009: 34). Esta influencia sobre un sujeto hace referencia a las intervenciones sociales. La política social procede a través de intervenciones, pero no toda intervención social es política social. En cada sociedad, las intervenciones sociales del Estado "amparan" grupos, cobijan sus intereses a través de acciones institucionalizadas, producen sus condiciones de vida y de reproducción.

El problema, tal como lo señalan los estudios de las dimensiones de género de las políticas sociales, es que las instituciones sociales construidas en torno a estos dos organizadores básicos (el mercado laboral y las conformaciones familiares), fallan en reconocer las asimetrías, reproduciendo el papel subordinado de las mujeres. Por el contrario, la ciudadanía basada en la igualdad de género supone una división sexual del trabajo más igualitaria en el mercado de trabajo y respecto de los cuidados domésticos. Ya el fundamento de una ciudadanía basada en el reconocimiento de la diferencia supone que autonomía y libertad de elección respecto de la reproducción y de las actividades de cuidados constituyen otras bases de derechos específicos de la mujer -derechos reproductivos, derecho a la socialización de los cuidados domésticos- (Draibe y Riesco, 2006). El abordaje del papel de las mujeres en el imaginario colectivo, merece una mirada que conjugue las necesidades de reconocimiento con las de redistribución (Fraser, 2000). Hay que destacar que la desigualdad en el mercado de trabajo se refleja en el sistema previsional, tanto en los países en que se han llevado a cabo reformas, como en los países en que estas no han ocurrido, lo que muestra una situación desventajosa para las mujeres que quedan fuera del sistema y reciben pensiones más bajas con las que deben vivir más tiempo (CEPAL, 2006b; Marco, 2004a y b).

Según Camila Arza (2014), en América Latina los sistemas de seguridad social contributivos de raíz bismarckiana no han logrado alcanzar una cobertura universal en la protección económica de los adultos mayores. Recientemente, varios países latinoamericanos impulsaron políticas orientadas a expandir la cobertura a través de prestaciones no contributivas o semicontributivas. Estas "pensiones básicas" son beneficios que no se encuentran estrictamente ligados a las contribuciones y al empleo formal y permiten extender la protección a una población más amplia.

Por otra parte, las desigualdades de género son otra limitación de los sistemas previsionales basados en el diseño bismarckiano. Arza (2013) plantea que en estos sistemas la unidad de protección es la familia, definida como un núcleo estable en el cual la mujer que se dedica al trabajo no remunerado del hogar (incluyendo cuidado de niños o ancianos) se encuentra protegida a través de su marido. El derecho a una pensión en caso de muerte es un ejemplo de esta cobertura "derivada" de la condición familiar. Uno de los problemas de este diseño es que responde cada vez menos a la realidad de la organización familiar actual. Por otro lado, a pesar de la tendencia en alza, aún hoy las mujeres siguen presentando tasas de participación en el 
mercado laboral menores que los hombres, por lo que a la hora de reclamar un beneficio jubilatorio contributivo las mujeres se encuentran en desventaja: acumulan menos aportes y por lo tanto muchas de ellas no logran alcanzar el mínimo requerido para obtener un beneficio; y las que sí lo obtienen, reciben beneficios más bajos, sea por haber contribuido pocos años o porque sus aportes y/o ingresos laborales fueron menores. Por ello, el sistema previsional resulta un espacio de interés para estudiar la persistencia o transformación de imaginarios que afectan la posición de las mujeres, en el marco de políticas con pretensiones de reconocimiento universal de derechos.

\section{Las desigualdades desde el Análisis crítico del discurso}

Los medios construyen realidades personales y grupales (Fairclough, 1995). Por lo que elanálisis de las noticias sobre las amas de casa beneficiarias de un haber previsional pondría de manifiesto los estereotipos y representaciones vigentes en la sociedad.

La construcción de realidades sociales en torno a ciertos gruposfundamenta las relaciones de convivencia entre segmentos de la población. En virtud de esta premisa, la formulación de implicaciones y la perpetuación de estereotipos genera igualmente algunos parámetros que orientan el cómo los individuos se relacionan y el cómo dichos individuos utilizan la lengua para promover sus relaciones sociales. La lengua refleja las instancias de poder y los niveles de relaciones sociales en la población. En consecuencia, la lengua refleja las consideraciones sobre el otro, el diferente y sobre los miembros asignados artificialmente a ciertos grupos sociales.

La lengua y el cómo se transmite el mensaje extienden la noción de contextos relacionales entre el mensaje real, el oculto y las intenciones entre los productores del discurso. De esta forma, los resultados de una investigación sobre el uso particular de la lengua revela el tratamiento que ciertos grupos dan a la población en general.

Como espejo de la sociedad, la lengua -por ende, los discursos- ayuda a que las comunidades reflexionen en torno a sus relaciones sociales en todos los niveles, sin diferenciar a los miembros de dichas comunidades ni a los actantes de dichas interacciones. Se ayuda a determinar cómo la manipulación de los hechos construye realidades en torno a ciertos grupos de la población.

El proveer evidencias concretas sobre el uso de la lengua escrita en las noticias permite concientizar a la población sobre el enfoque que ciertos periódicos usan para reproducir hechos sociales que envuelven a las minorías y permite otorgarherramientas para una lectura crítica de las noticias.

Entonces, hablar de Análisis crítico del discurso, implica tener claro qué es el discurso mismo: un mensaje escrito y/o hablado que da cuenta de ideas, principios, conocimientos y que: "existe 
cuando tomamos varios elementos y los articulamos a nuestro antojo y conveniencia para enviar un mensaje. Por lo tanto los discursos son siempre intencionales, es decir, llevan una intención, son portadores de un mensaje previamente concebido y elaborado por el comunicador o emisor". (Instituto Centroamericano de Estudios Políticos, 2004: 15). Ahora bien, los discursos no existen aislados, no existen con independencia de otros, "nunca un discurso existe por sí mismo sin estar anclado en algún otro" (Iníguez, 2006: 113). Ciertamente, entre los discursos se da una interrelación, una "intertextualidad". Por otra parte, todo enunciado, todo discurso, está históricamente condicionado, determinado. "El discurso es lenguaje como práctica social determinada por estructuras sociales" (Iníguez, 2006: 126). Lo que significa que: el lenguaje es una parte de la sociedad y no algo externo a ella; es un proceso social; es un proceso condicionado socialmente, históricamente, en el mismo sentido que lo son otras partes o procesos no lingüísticos (Iníguez, 2006: 126).

El Análisis del discurso "es un campo de estudio nuevo, interdisciplinario, que ha surgido a partir de algunas otras disciplinas de las humanidades y de las ciencias sociales, como la lingüística, los estudios literarios, la antropología, la semiótica, la sociología y la comunicación oral" (Van Dijk, 1990: 35). Es una disciplina que se interesa por los contextos del discurso, los procesos cognitivos de la producción y la recepción del mismo, y por los contextos socioculturales del uso de la comunicación. Su principal objetivo es producir descripciones del discurso en un texto y contextos determinados (contexto cognitivo, social, cultural o histórico).

En cuanto a su relación con la sociedad y la cultura, Van Dijk plantea que "sólo podemos comprender la importancia del discurso en los procesos sociales y en las relaciones de poder contemporáneos si reconocemos que el discurso constituye a la sociedad y a la cultura, así como es constituido por ellas" (2005: 399).

Por lo demás, "lo característico del Análisis Crítico del Discurso es que toma partido a favor de los grupos oprimidos en contra de los grupos dominantes, y que manifiesta abiertamente la vocación emancipadora que la motiva" (Fairclough y Wodak, 2005: 368). El ACD se ocupa de problemas sociales y de cuestiones políticas. Del poder social de grupos y/o instituciones. Del control del discurso (político, académico, público, corporativo) por parte de clases dominantes. Analiza la reproducción del dominio y la desigualdad social y la resistencia contra ellas. Obsérvese con todo que el discurso no es sólo un recurso más entre otros: quienes controlan el discurso público controlan ampliamente la mentalidad social e indirectamente la acción pública; y, por consiguiente, controlan también la estructura social (Van Dijk, 1999: 34).

En concreto, el Análisis Crítico del Discurso se dirige más hacia los problemas o los temas que hacia los paradigmas (Van Dijk, 1997: 15). Enfoca su atención en problemas sociales tales como el sexismo, el racismo, el colonialismo, etcétera; asimismo, se centra en las relaciones de poder, dominación y desigualdad, así como en la reproducción u oposición (resistencia) a través del texto y del habla. 
Esta investigación tiene carácter novedoso debido a que no se han realizado aún análisis discursivos en torno a las representaciones mediáticas de la figura del ama de casa dentro del sistema previsional argentino y los efectos que la difusión de estas trajeron en el arraigo de ciertos imaginarios sobre la valoración económica, social, cultural, política y comunicacional de la labor de la ama de casa dentro del mercado.

Es importante analizar la perspectiva de los acontecimientos que difunden los medios de comunicación, ya que son estos los que coadyuvan a la reproducción de las ideologías dominantes: "Los medios informativos no describen pasivamente ni registran los sucesos noticiables del mundo, sino que los (re)construyen activamente, basándose, mayoritariamente, en muchos tipos de discurso de fuente. Los intereses corporativos, los valores de la información, las rutinas institucionales, las ideologías profesionales y los formatos esquemáticos de las noticias desempeñan un papel importante en esta transformación" (Van Dijk, 1997: 78).

Por ello, en esta investigación se vio la necesidad de articular un análisis crítico del discurso en torno a las noticias publicadas sobre las amas de casa y el Plan de Inclusión Previsional, tomando en cuenta el contexto cognitivo, social, cultural e histórico en el que fueron difundidas. En este sentido, se tiene en cuenta que "el Análisis Crítico del Discurso proporciona las herramientas teóricas y metodológicas necesarias para un enfoque crítico fundamentado del estudio de los problemas sociales, el poder y la desigualdad" (Van Dijk, 2006: 62).

Cabe aclarar que no existe un único método o manera de hacer análisis crítico del discurso. Algo que se puede generalizar es que la colección de datos no se separa estrictamente del análisis, sino que puede continuar después del análisis primario donde se determinan los temas y se encuentran los elementos necesarios para la conceptualización de ciertos factores centrales del uso desigual de poder (Meyer, 2003: 49).

\section{Metodología y selección del corpus documental}

El corpus de esta investigación es el resultado de un trabajo de recopilación de todas aquellas notas de prensa de tres diarios de tirada nacional (Página/12, Clarín, La Nación) relativas a acciones en materia previsional relacionadas con el Plan de Inclusión Previsional entre el 10 de diciembre de 2004 -promulgación de la Ley 25994, con la que entra en vigencia la moratoria previsional que permitía las jubilaciones sin aportes previsionales completos- y el 30 de abril 2007, fecha en que vence la moratoria. El monitoreo se hizo en 840 ediciones, de las cuales se extrajeron 15 notas de Clarín; 21 notas en La Nación y 24 notas en Página/12. Cada nota recopilada permitió identificar el discurso de los actores involucrados; la forma de presentación 
de estas, junto con las notas de opinión y las columnas editoriales permitieron identificar el discurso mediático en torno al tema.

La muestra ha sido definida en función de criterios de representatividad cualitativos. Según Irene Vasilachis, la investigación cualitativa es interpretativa, hermenéutica e inductiva y utiliza métodos de análisis sensibles a las personas estudiadas y al contexto social en el que los datos son producidos, a lo que complementa que "es relacional, se sustenta fundamentalmente, en la comunicación" (2006: 8). La cantidad de notas recogidas ha sido determinada en función a la saturación de la información dada por las mismas, entendiéndose que "Los criterios para determinar la saturación son la combinación de los límites empíricos de los datos, la integración y la densidad de la teoría y la sensibilidad teórica del analista" (Glaser y Strauss, 1965: 61).

Debido que se pretende estudiar cómo los medios gráficos nacionales construyeron diversas representaciones mediáticas del Plan de Inclusión Previsional, a partir de la inserción del ama de casa como beneficiaria directa, nuestros presupuestos epistemológicos de partida son resultados de las aportaciones del análisis crítico del discurso. Por la investigación propuesta, estimamos que la metodología adecuada para el marco de análisis teórico es el análisis crítico por la trilogía conceptual poder-discurso-ideología que lo sustenta. A pesar de esto, cabe aclarar que atendiendo a los intereses de este artículo, no nos centraremos en el "qué" se comunica sino en el "cómo" se lo hace. Estas investigaciones con enfoque de género son importantes, ya que como lo explica Sandra Chahen, "uno de los aspectos más importantes para el análisis fue la imagen que la sociedad construía de las mujeres, y allí se volvieron cruciales los medios masivos, considerados una instancia fundamental de socialización junto con la familia y la escuela" (2007: 95).

La metodología del análisis crítico del discurso consiste en la identificación de los participantes y la limitación del enfoque a la acción más interesante desde el punto de vista del investigador. Es necesario aclarar que no existe un único método o manera de hacer análisis crítico.

Cabe aclarar que el modelo de análisis crítico del discurso que se usa en este análisis es el del método histórico discursivo, que tiene como principal exponente a Ruth Wodak, quien plantea un estudio basado en el análisis del impacto de la ideología en cuestiones como el género, el nacionalismo o la inmigración. El análisis de Wodak parte de la base lingüística para ahondar en una crítica sociolingüística, que permita develar el modo en que determinadas prácticas discursivas pueden funcionar ideológicamente para favorecer la hegemonía de un grupo sobre otro; y por otra parte, comprender mejor cómo se percibe la representación de la dominación por parte de los dominados.

Con este artículo, entonces, se pretende conocer cómo se construyeron y difundieron discursos sobre las amas de casa como beneficiarias de un haber previsional. En otras palabras, se 
busca identificar cómo se conjugaron los imaginarios subyacentes de la sociedad con la incorporación del ama de casa al sistema de previsión social a partir de esta medida.

\section{Algunos resultados}

Como se mencionó en el apartado anterior, esta investigación tiene como corpus el resultado de un trabajo de recopilación de todas aquellas notas de prensa de tres diarios de tirada nacional (Página/12, Clarín, La Nación) que tienen como foco noticioso lo relacionado al Plan de Inclusión Previsional.

El procesamiento total de las notas abordadas da cuenta que, en los años seleccionados para el análisis, las representaciones de la "meritocracia" en relación a la inclusión del ama de casa en el Sistema Previsional Argentino ha tenido distintos espacios en los discursos de los medios mencionados, pero sin darle un tratamiento pormenorizado a la situación. Sólo se encontraron sesenta artículos en los medios gráficos mencionados y en el periodo de tiempo detallado.

Tal como puede inferirse, el desarrollo de esta política pública no tuvo un espacio notorio en las agendas de estos medios, ya que la publicación de notas sobre el tema es esporádica y, por lo general, el factor de "noticiabilidad" estuvo dado en la cercanía a los años electorales.

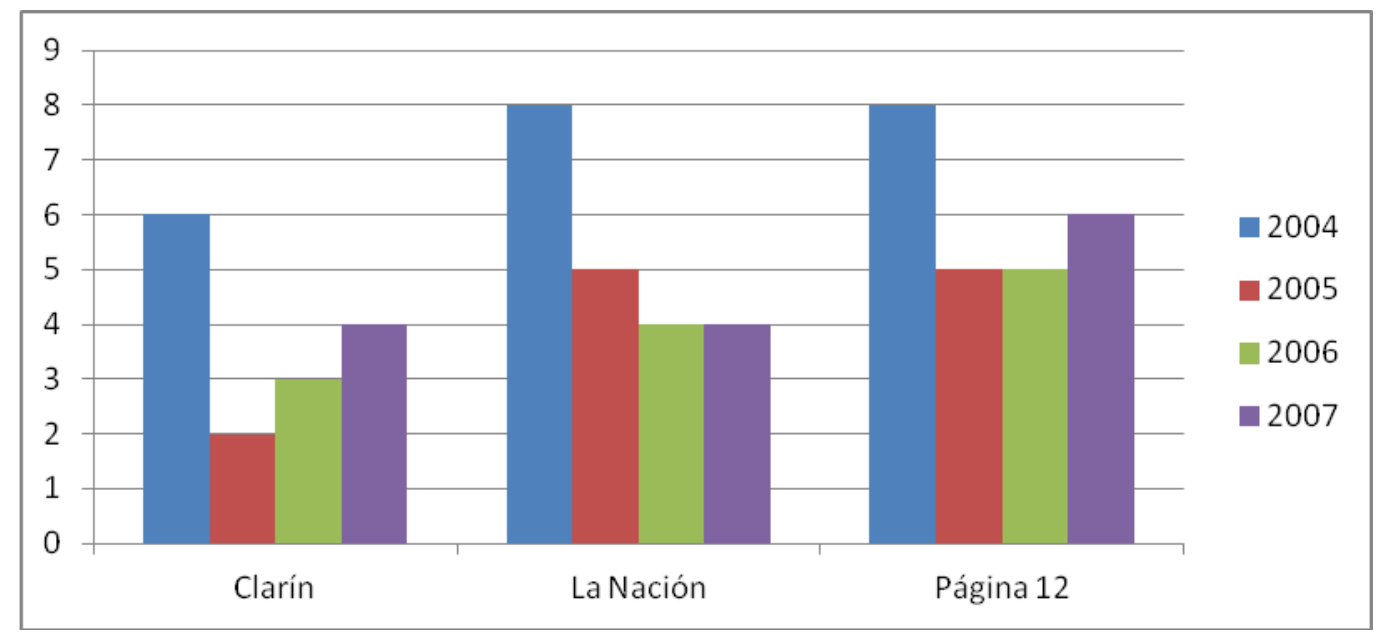

Figura 1: Cantidad de notas referidas al Plan de Inclusión Previsional y la inserción del ama de casa como beneficiaria directa. Elaboración propia.

Cada nota recopilada permitió identificar el discurso de los actores involucrados, así como la forma de presentación de estas, junto con las notas de opinión y columnas editoriales que permitieron identificar el discurso mediático en torno al tema.

Tanto en Clarín como en La Nación, las notas referidas al Plan de Inclusión Previsional y la inserción del ama de casa en este plan, aparecen en editoriales o en columnas. En la mayoría 
de los casos, sin fuentes consultadas o -en el caso que existiesen- las fuentes eran hombres 0 jubilados varones del SIPA, demostrando una clara impronta conservadora.

En Página/12, por el contrario, la mayoría de las fuentes consultadas fueron mujeres jubiladas a través del PIP, transformándolas en interlocutoras válidas al legitimar de este modo sus voces. Además, la mayoría de las notas se publicaron en el suplemento Las 12 y fueron escritas por periodistas con una clara visión de género, como Luciana Peker o Marta Dillon.

En cuanto al contenido de las notas, La Nación puso el foco en el "vaciamiento del ANSES", producto de la inclusión del ama de casa y de los autónomos al sistema previsional con titulares como "Pan para hoy, hambre para mañana" (1 de abril de 2006); "Una fiesta cara y peligrosa" (11 de mayo de 2006); "Los jubilados también suman votos" (10 de septiembre de 2006); "La medida más cara" (10 de mayo de 2006), entre otros.

Clarín, por su parte, titulaba "El 50\% de los jubilados no aportó y se financia con la inmovilidad de los aportares" (2 de agosto de 2007); "La caja de los jubilados sigue sangrando", "La trampa de los subsidios" (13 de julio de 2004); "Las mujeres y la jubilación, ¿una relación con futuro?" (10 de diciembre de 2005), entre otros.

Las valoraciones adjudicadas a esta política pública son principalmente negativas, relacionadas al "no trabajo" y "clientelismo", sin siquiera hacer un análisis de los cambios que significan estas medidas en las personas beneficiadas. En general, la línea editorial presente se abocaba a expresar con sutileza -y a veces no tanto- el fastidio por la inclusión de este tipo de medidas que arremetían un beneficio universal. En particular, estas notas tienen un alto contenido tendencioso y crítico hacia aquellos/as que no se desempeñaron en el mercado formal del trabajo.

Es curioso notar que estas noticias fueron publicadas durante la primera moratoria previsional. En la segunda, el tema no fue tocado prácticamente en los medios mencionados. En relación a esto, en el análisis percibimos que el discurso implícito prevaleciente en los artículos fue el de "las amas de casa se jubilan sin trabajar", dejando en manifiesto la postura patriarcalizada de estos medios en la construcción del relato.

Por su parte, en Página/12 sucedió lo contrario. Los discursos difundidos tienen mensajes empáticos hacia los trabajadores cuentapropistas, autónomos y amas de casa. Esto puede apreciarse en titulares como "Con la moratoria se arrimó el bochín" (12 de junio de 2007); "El gobierno gasta más en obras y en jubilados" (27 de marzo de 2007); "Una moratoria que pegó en el blanco" (7 de abril de 2007); "Los motivos para el crecimiento del consumo de los sectores bajos" (22 de abril de 2007).

Todas las notas publicadas por este medio tienen un alto contenido de análisis que, en muchas ocasiones, abarcaba doble página o una página completa. Un punto clave a destacar es que sólo en Página/12 hay fuentes estadísticas y fuentes académicas. 
Clarín y La Nación construyen un prodestinatario que se manifiesta como el "yo trabajador formal", cuyo sentido de pertenencia al mercado laboral es propio de la clase media. En esta línea, como destinatario positivo es posible mencionar a aquellas familias o personajes públicos cuya ideología sea de corte conservador. Lo opuesto sucede con Página/12, que como prodestinatario se manifiesta el "obrero", cuyo sentido de pertenencia al mercado laboral es propio de la clase baja "popular", cuya trayectoria laboral ha sido intermitente. En conjunción con esta línea, como destinatario positivo se pueden mencionar movimientos sindicales y feministas, que promulgan la emancipación de las mujeres.

Es oportuno resaltar que, mientras que las percepciones negativas en la prensa hegemónica nacional estuvieron dadas en el Plan de Inclusión Previsional como política que buscaba la universalidad de haberes previsionales en la población anciana, en la prensa contrahegemónica, las impresiones negativas estuvieron dadas en la precarización de los mercados laborales durante los gobiernos neoliberales, que conllevaron a la instauración de este tipo de medidas.

\section{Reflexiones finales}

Las representaciones en torno a la inclusión del ama de casa al Plan de Inclusión Previsional ¿surgieron a partir del mensaje instaurado en los medios o fue la sociedad la que impuso la agenda y el modo de tratar este tema?

A lo largo de este artículo se ha pretendido exponer cómo los distintos medios gráficos construyeron y difundieron discursos y representaciones de las amas de casa incluidas bajo la figura de no aportantes en función a su línea editorial. Para poder caracterizarlos se realizó un análisis profundo de los discursos difundidos por tres diarios de tirada nacional: Página/12, Clarín y La Nación. En este punto donde converge el análisis se constituye el auténtico objeto de nuestro interés. A lo largo de la interpretación de los discursos fue posible observar cómo en cada nota se hizo - de modo sutil- una evaluación moral acerca de la "meritocracia", en torno al otorgamiento de beneficios previsionales a las ancianas amas de casa. Es preciso recalcar el modo en que cada medio ha creado distintas versiones de amas de casa: por un lado, aquel que invisibiliza el trabajo reproductivo no remunerado; por otro, aquel que ve al ama de casa como sujeta merecedora de derechos previsionales.

Un punto a recalcar es que se identificaron coincidencias y afinidades en la construcción de la realidad y de las sujetas por parte de Clarín y La Nación; mientras que en Página/12 encontramos la antítesis.

Los planteos presentados por los medios llamados hegemónicos continúan desvalorizando el trabajo realizado por las amas de casa al interior de los hogares, por no encuadrarse en el 
ámbito del mercado laboral y, en sintonía, naturalizando esta labor como un natural acto de amor y entrega por parte de las mujeres. Como visión contra hegemónica, Página/12 erige un posicionamiento desde la identificación con la clase subordinada, con aquellos/as desvalidos, olvidados, no escuchados.

Jesús Martín-Barbero resume este reflejo social al especificar que

\begin{abstract}
a mayor cantidad de información, menos sentido. Cada día estamos informados de más cosas pero cada día sabemos menos qué significan. ¿Cuánta de la enorme cantidad de información que recibimos sobre el país y el mundo se traduce en mayor conocimiento de los otros, en posibilidades de comunicación y en capacidad de actuartransformadoramente sobre nuestra sociedad? Tenemos información pero se nos escapa el sentido, vivimos en la euforia de una participación que la vida misma se encarga de mostrarnos lo que tiene de simulacro (2000: 7).
\end{abstract}

A pesar de las distintas políticas públicas y el llamado a concientización en cuanto a la correcta y equitativa división sexual del trabajo, el imaginario colectivo aún tiene marcado a fuego el supuesto de que las mujeres son aquellas personas dedicadas exclusivamente al cuidado de la familia, lejos de su desarrollo como ciudadanas, con iguales derechos que los hombres, niños y niñas. Su invisibilización aún es latente, su ausencia como beneficiarias directas de programas y políticas sociales es abrumadora. Ante esto, en muchos casos, los medios amplifican estas realidades vedadas.

\title{
Bibliografía
}

Benveniste, E. (1978). Problemas de Lingüística General, Tomo II. México: Siglo XXI.

Bolívar, A. (2007). Análisis del discurso, ¿por qué y para qué? Venezuela: Editorial CEC. Recuperado

https://www.google.com.ar/search?hl=es\&tbo=p\&tbm=bks\&q=isbn:980388350X

Bourdieu, P. (1991). El sentido práctico. Argentina: Siglo XXI Editores. Recuperado dehttps://sociologiaycultura.files.wordpress.com/2014/02/bourdieu-el-sentidoprc3a1ctico.pdf

Chaher, S. (2007). Primeras aproximaciones al periodismo de género. En Santoro, S. y Chaher S. (comps.). Las palabras tienen sexo. Introducción a un periodismo con perspectiva de género. Buenos Aires: Artemisa Comunicación Ediciones.

Di Costa, V. (2011). Plan de Inclusión Previsional en Argentina: alcances y desafíos en su impacto redistributivo. Estudios Sociales Contemporáneos, 5/6, pp. 137-151. 
Draibe, S. y Riesco, M. (2006). Estado de Bienestar, desarrollo económico y ciudadanía: algunas lecciones de la literatura contemporánea, Serie Estudios y Perspectivas 55. México: CEPAL.

Carbó, T (2001). El cuerpo herido o la constitución del corpus en análisis del discurso. Escritos, 23, pp. 17-47. Recuperado de http://www.buap.mx/portal_pprd/work/sites/escritos/resources/LocalContent/31/1/carbo. pdf

Fairclough, N. (2003). El análisis crítico del discurso como método para la investigación en ciencias sociales. En R. Wodak y M. Meyer (Comps.). Métodos de Análisis Crítico del Discurso (pp. 179- 203). Barcelona: Gedisa.

Foucault, M. (1973). El orden del discurso. Ciudad Autónoma de Buenos Aires, Argentina: Tusquets editores.

Jäger, S. (2003). Discurso y conocimiento: aspectos teóricos y metodológicos de la crítica del discurso y del análisis de dispositivos. En Wodak, R. y Meyer, M. Métodos de análisis crítico del discurso. Barcelona: Gedisa

Lippmann, W. (1964). Public opinión, 10 dehttp://www.gutenberg.org/ebooks/6456

Orloff, A. S. (1993). Gender and the social rights or citizenship: the comparative analysis of gender relations and welfare states. Recuperado de http://www.people.fas.harvard.edu/ iversen/PDFfiles/Orloff1993.pdf

Pereyra, F. (2012). La regulación laboral de las trabajadoras domésticas en Argentina: situación actual y perspectivas. En Esquivel, V.; Faur, E. y Jelin, E. (editoras). Las lógicas del cuidado infantil. Entre las familias, el Estado y el mercado. Buenos Aires: IDES.

Picchio, A. (1992). Social reproduction: the political economy of the labour market. Cambridge: Cambridge University Press.

Picchio, A. (1994). El trabajo de reproducción, tema central en el análisis del mercado laboral. En Borderías, C.; Carrasco, C. y Alemany, C. (comps). Las mujeres y el trabajo. Recuperado de http://www.ccee.edu.uy/ensenian/catgenyeco/Materiales/2011-0810\%20M3\%20-\%20Picchio

Picchio, A. (1999). Visibilidad analítica y política del trabajo de reproducción social. En Carrasco, C. (ed). Mujeres y economía. Barcelona: Icaria-Antrazyt.

Picchio, A. (2001). Un enfoque macroeconómico ampliado de las condiciones de vida. Conferencia Inaugural de las Jornadas "Tiempos, trabajos y género", Universidad de Barcelona, España.

Razavi, S. (2007). The Political and Social Economy of Care in a Development Context. Conceptual Issues, Research Questions and Policy Options. Recuperado de 
http://www.unrisd.org/80256B3C005BCCF9/(httpAuxPages)/2DBE6A93350A7783C125 73240036D5A0/\$file/Razavi-paper.pdf

Rodriguez Enríquez, C. (2007). Economía del cuidado, equidad de género y nuevo orden económico internacional. Recuperado de http://bibliotecavirtual.clacso.org.ar/ar/libros/sursur/giron_correa/22RodriguezE.pdf

Rodríguez Enríquez, C. (2011). Programas de Transferencias Condicionadas de Ingreso e Igualdad de Género. ¿Por dónde anda América Latina? Santiago: CEPAL, Serie Mujer y Desarrollo 109.

Rodríguez Enríquez, C. (2012). La cuestión del cuidado ¿el eslabón perdido del análisis económico? Revista Cepal, 109.

Santander Molina, P. (2009). Por qué, para qué y cómo hacer Análisis del Discurso de los medios de comunicación. En Santander Molina (Ed.). Analizando los medios y la comunicación teoría y métodos. Valparaíso, Chile: Ediciones Universitarias de Valparaíso. Recuperado de http://www.portalcomunicacion.com/pdf/analizando.pdf

Van Dijk, T (1990). La noticia como discurso, comprensión, estructura y producción de la información. Barcelona, España: Paidós. Recuperado de https://linguistica2013.files.wordpress.com/2013/11/van-dijk-teun-la-noticia-comodiscurso.pdf

Van Dijk, T (1999). El análisis crítico del discurso. Anthropos, 186, pp. 23-36. Recuperado de http://www.discursos.org/oldarticles/El\%20an\%E1lisis\%20cr\%EDtico\%20del\%20discurs o.pdf

Van Dijk, T (2003). Ideología y análisis del discurso. Utopía y Praxis Latinoamericana. Revista Internacional de Filosofía Iberoamericana y Teoría Social, 29, pp. 9-36. Recuperado de http://www.discursos.org/oldarticles/ldeolog\%EDa\%20y\%20an\%E1lisis\%20del\%20disc urso.pdf

Van Dijk, T. (2009). Discurso y poder. Contribuciones a los Estudios Críticos del Discurso. Barcelona: Gedisa.

Vasilachis de Gialdino, I. (coord.) (2006). Estrategias de investigación cualitativa. Barcelona: Gedisa.

Verón, E. (1968). Conducta, estructura y comunicación. Buenos Aires: Editorial Jorge Alvarez.

Wodak, R. y Meyer, M. (2003). Métodos de análisis crítico del discurso. Barcelona: Gedisa. 